\title{
Upper and lower bounds for the waiting time in the symmetric shortest queue system
}

\author{
Ivo Adan, Geert-Jan van Houtum and Jan van der Wal \\ Eindhoven University of Technology, Eindhoven, The Netherlands
}

\begin{abstract}
In this paper we compare the exponential symmetric shortest queue system with two related systems: the shortest queue system with Threshold Jockeying and the shortest queue system with Threshold Blocking. The latter two systems are easier to analyse and are shown to give tight lower and upper bounds respectively for the mean waiting time in the shortest queue system. The approach also gives bounds for the distribution of the total number of jobs in the system.
\end{abstract}

\section{Introduction}

In job shop like production systems, flexible manufacturing systems and computer and communication networks with parallel server stations, one might have to assign the jobs to a specific server immediately upon the time of arrival. Then a natural strategy is to assign an arriving job to the server with the shortest queue. However, the impact of the shortest queue assignment strategy on the system behaviour is not intuitively clear. Therefore, methods are being developed to evaluate its performance. In this paper we present an approach to efficiently calculate the mean waiting time for shortest queue routing at a single station.

The symmetric shortest queue system with two queues, Poisson arrivals and exponential service times has been extensively studied in the literature. Haight [15] introduced the problem. Kingman [18] and Flatto and McKean [10] use a uniformization technique to determine the generating function of the stationary queue length distribution. Another analytic approach is given in Cohen and Boxma [4] and Fayolle and Iasnogorodski [8]. They show that the analysis of the symmetric shortest queue system can be reduced to that of a Riemann-Hilbert boundary value problem. Though mathematically elegant, these analytical results offer limited practical means for computing performance characteristics. Recently, it has been shown that the symmetric shortest queue problem can be solved completely by the compensation approach introduced in Adan et al. [1]. The advantage of this approach is that the analytical results are easily exploited for numerical computations. 
Many numerical studies have appeared on the present problem. Most studies, however, deal with the evaluation of approximating models, see e.g. Gertsbakh [12], Grassmann [13], Rao and Posner [24] and Conolly [5]. Using linear programming, Halfin [16] obtains upper and lower bounds for the mean and the distribution of the number of jobs in the system. Heavy traffic diffusion approximations can be found in Foschini and Salz [11]. Knessl et al. [19] derive asymptotic expressions for the stationary queue length distribution. Zhao and Grassmann [14] exploit some results of Flatto and McKean [9] to derive a numerically stable algorithm for computing the queue length probabilities. Schassberger $[25,26]$ uses an iterative method to numerically obtain approximating values for the queue length probabilities. The studies mentioned are all restricted to systems with two parallel queues.

For more than two queues no analytical results are available. Hooghiemstra et al. [17] develop a power series method to calculate the stationary queue length distribution for fairly general multidimensional exponential queueing systems. Their method is not restricted to systems with two queues, but applies equally well to systems with more queues. As far as the shortest queue system is concerned, Blanc [3] reports that the power series method is numerically satisfactory for practically all values of the workload for systems with up to 10 parallel queues. However, the theoretical foundation of this method is still incomplete. Nelson and Philips [21] derive an approximation for the mean response time for the shortest queue system with multiple queues. They report that their approximation has a relative error of less than 2 percent for systems with at most 16 queues and with service utilizations over the range from 0 to 0.99 . A common disadvantage of the numerical methods mentioned is that in general no error bounds can be given.

In this paper we derive upper and lower bounds for the mean waiting time in the shortest queue system by comparing it with two other queueing models which are easier to evaluate. These two models exploit the aspect that in the shortest queue system high unbalance in queue lengths is very unlikely.

The model that produces the lower bound for the waiting time is the shortest queue system with Threshold Jockeying, i.e. if the difference between the longest queue and the shortest queue exceeds a certain threshold, then one customer moves from the longest queue to the shortest queue.

This will improve the performance of the system somewhat because the situation in which one server is idle while there are waiting customers in another queue is less likely to occur. Note that for a threshold of 1 the system behaves as an $M / M / N$ queue with $N$ the number of servers.

The unbalance in the queue lengths can only occur if temporarily the servicing in one queue goes so much faster than the servicing in another queue that even sending all arrivals to the shorter queue cannot compensate it. Therefore one might expect that for a somewhat larger threshold ( 3 or 4 maybe) the mean waiting time will be almost identical to the one in the original system. 
A model giving an upper bound is the model with Threshold Blocking. In this model the server in the shortest queue is switched off as soon as the difference between the longest and the shortest queue reaches a certain threshold. If the difference drops below the threshold the servicing is resumed. It seems clear that blocking a server will have a negative impact on the performance of the system. And, as before, the larger the threshold is taken, the less blocking one should get and the better the bound should be.

We will prove that these two queueing models indeed provide lower and upper bounds for the mean waiting time in the shortest queue system. Even more, we will see that the total number of jobs in the system is stochastically smaller and larger, respectively, than in the original shortest queue system.

The line of proof is similar to the ones in Van der Wal [28], Van Dijk and Van der Wal [7] and Van Dijk and Lamond [6]. First of all we note that the three models lead to Markov processes that are equivalent to Markov chains. The mean performance characteristics of these Markov chains are the same as the ones of the Markov processes. In order to compare the Markov chains we look at finite period costs. By induction we show one model to be superior to another for each finite number of periods. Letting the number of periods go to infinity then yields the result for the average performance. We will only give the proofs for the case of two queues. For more than two queues the proofs are essentially the same but notationally more complex.

Alternatively, the shortest queue problem can be approximated by the common queue system, i.e. the $M / M / N$ queue, or by treating all queues as bounded. In the latter approximation arriving jobs finding all buffers full are rejected (and thus receive zero waiting times). Numerical results show that these simple approximations perform poorly compared to the use of the upper and lower bounds. There are several possibilities to improve these simple approximations. However, more sophisticated approximations still have the disadvantage that no error bounds can be given.

A generalization of the symmetric shortest queue problem has been studied by Lui and Muntz [20]. They consider the problem with non-identical exponential servers and the, in that case more sensible, shortest expected delay routing. In case of identical servers this problem reduces to the shortest queue problem. Lui and Muntz also construct two queueing systems providing upper and lower bounds for the mean response time in the shortest expected delay problem. Their upper bound model is based on Threshold Blocking and a second mechanism using artificial capacity constraints. This second mechanism also has a negative impact on the performance of the system. Their lower bound model is not related to the Threshold Jockeying model. For the special case of symmetric queues the bounds presented by Lui and Muntz are less effective than the ones presented in this paper in the sense that the same accuracy is obtained with higher computational costs.

In section 2 we discuss the translation of the Markov processes into the 
equivalent Markov chains. Section 3 discusses the technique of comparing the finite period models. Section 4 deals with two essential monotonicity results for the original shortest queue system: less customers in the system lead to lower costs, and a balanced system performs better than an unbalanced one. In sections 5 and 6 we prove that the models with Threshold Jockeying and Threshold Blocking lead to lower and upper bounds on the mean waiting time. In section 7 we consider the stochastic monotonicity of the total number of jobs in the three systems. In section 8 it is shown how these two queueing models can be analyzed by using the matrixgeometric approach developed by Neuts [22]. Section 9 provides numerical results showing that the mean waiting time in shortest queue systems with up to 50 parallel queues can be obtained from the two other systems using very moderate threshold values. In section 10 we compare the use of bounds with that of simple truncation. Section 11 is devoted to some conclusions.

\section{Markov processes and Markov chains}

In all three Markov processes jobs arrive according to a Poisson process with rate $\lambda$. Immediately upon arrival a job is sent to the shortest queue. All service times are exponential with rate $\mu$. The state of the Markov process is a vector $s=\left(s_{1}, \cdots, s_{N}\right)$ with $N$ the number of servers. Exploiting the symmetry in the model we may assume that $s_{1} \geq \cdots \geq s_{N}$. So $s_{1}$ is the number of jobs in the longest queue, $s_{2}$ is the number of jobs in the second longest queue and so on. The maximal output rate from a state is $\lambda+N \mu$. Without loss of generality we may take $\lambda+N \mu=1$.

The original shortest queue system and the model with Threshold Jockeying are ergodic if $N \mu>\lambda$. For the model with Threshold Blocking this condition is not sufficient, because blocking reduces the service capacity. The condition under which the model with Threshold Blocking is ergodic will be formulated in section 7 .

Let $Q$ be the generator of one of the three Markov processes that we are dealing with, then the corresponding equilibrium distribution $p$ satisfies $p Q=0$. Instead of studying the Markov process with generator $Q$ we look at the Markov chain with transition matrix $P=I+Q$. Recall that $\lambda+N \mu=1$, so $P$ is indeed a stochastic matrix. Clearly, the equilibrium distribution $p$ of the Markov process is the same as the one for the Markov chain. Also, mean costs in the Markov process and the Markov chain are easy to compare. If $c(s)$ is the cost rate in the Markov process and we let $c(s)$ be the costs per period in the Markov chain, then the Markov process and the Markov chain will have the same average costs: $\Sigma_{s} p(s) c(s)$.

From now on we only consider the three Markov chains.

\section{Finite period models}

For the basic shortest queue system we define $v_{n}(s)$ as the expected $n$-period costs when starting in state $s$. Similarly $u_{n}$ and $w_{n}$ denote the expected $n$-period costs 
in the model with Threshold Jockeying and Threshold Blocking. Defining $u_{0}=v_{0}$ $=w_{0}=0$, we will try to prove by induction that for all (relevant) states $s$ and for all $n$

$$
u_{n}(s) \leq v_{n}(s) \leq w_{n}(s)
$$

Of course, this implies that the average costs for the three models are ordered in the same way.

If the cost function is the total number of jobs in the system, then we can conclude that the average numbers of jobs in the system for the three models are ordered. And by Little's formula the same holds for the mean waiting times.

If the costs are 1 if the total number of jobs in the system exceeds $M$ and 0 otherwise, and the ordering result holds for all $M$, then the total numbers of jobs in the systems are stochastically ordered.

In order to be able to prove (1) we first have to establish some monotonicity results for the functions $v_{n}$.

\section{Monotonicity of the functions $v_{\mathrm{n}}$}

From hereon we will consider the case of two queues only. The results hold for $N \geq 3$ as well, but the notations become more complex.

Because of the symmetry we only have to consider states $s=(i+l, i)$ with nonnegative integers $i$ and $l$.

As cost function we use the total number of jobs in the system, so $c(i+l, i)=2 i+l$. The monotonicity results that we need are the following intuitively obvious inequalities:

LEMMA 1

For all $n \geq 0$ we have

$$
\begin{aligned}
v_{n}(i+l, i) \geq v_{n}(i+l-1, i+1), & i \geq 0, l \geq 2, \\
v_{n}(i+l+1, i) \geq v_{n}(i+l, i), & i \geq 0, l \geq 0 \\
v_{n}(i+l, i+1) \geq v_{n}(i+l, i), & i \geq 0, l \geq 1 .
\end{aligned}
$$

So (2) states that more balance at the beginning leads to lower costs. Inequalities (3) and (4) say that removal of a job from the system reduces the expected costs.

\section{Proof of lemma 1}

The proof will be given by induction. Since $v_{0}=0$ inequalities (2)-(4) trivially hold for $n=0$. Assuming (2) (4) to hold for $n$ we will establish them for $n+1$. In order to prove this lemma we will distinguish a number of cases. 


\section{Proof of (2)}

Case a: $l \geq 3$.

We have

$$
\begin{aligned}
v_{n+1}(i+l, i)= & 2 i+l+\lambda v_{n}(i+l, i+1) \\
& +\mu v_{n}(i+l-1, i)+\mu v_{n}\left(i+l,(i-1)^{+}\right),
\end{aligned}
$$

where $(i-1)^{+}$denotes $\max (0, i-1)$, and

$$
\begin{aligned}
v_{n+1}(i+l-1, i+1)= & 2 i+l+\lambda v_{n}(i+l-1, i+2) \\
& +\mu v_{n}(i+l-2, i+1)+\mu v_{n}(i+l-1, i) .
\end{aligned}
$$

Now compare the right hand sides (RHS) of (5a) and (5b). The first terms are equal, the second term in (5a) is at least equal to the second one in (5b) because of (2), similarly the third and fourth terms are ordered. For the fourth term the case $i=0$ follows from (3). So $v_{n+1}(i+l, i) \geq v_{n+1}(i+l-1, i+1)$.

Case b: $l=2$.

$$
\begin{gathered}
v_{n+1}(i+2, i)=2 i+2+\lambda v_{n}(i+2, i+1) \\
+\mu v_{n}(i+1, i)+\mu v_{n}\left(i+2,(i-1)^{+}\right), \\
v_{n+1}(i+1, i+1)=2 i+2+\lambda v_{n}(i+2, i+1)+\mu v_{n}(i+1, i)+\mu v_{n}(i+1, i) .
\end{gathered}
$$

The first, second and third terms on the RHS are equal, the fourth terms are ordered because of (2) if $i>0$ or (3) if $i=0$.

So $v_{n+1}(i+2, i) \geq v_{n+1}(i+1, i+1)$.

Proof of (3)

Case a: $l \geq 1$.

$$
\begin{array}{rl}
v_{n+1}(i+l+1, i)=2 & i+l+1+\lambda v_{n}(i+l+1, i+1) \\
& +\mu v_{n}(i+l, i)+\mu v_{n}\left(i+l+1,(i-1)^{+}\right), \\
v_{n+1}(i+l, i)=2 & i+l+\lambda v_{n}(i+l, i+1) \\
& +\mu v_{n}(i+l-1, i)+\mu v_{n}\left(i+l,(i-1)^{+}\right) .
\end{array}
$$

The first term on the RHS of (7a) is larger than the one of (7b), the other three terms are ordered by (3).

So $v_{n+1}(i+l+1, i) \geq v_{n+1}(i+l, i)$. 
Case b: $l=0$.

$$
\begin{aligned}
v_{n+1}(i+1, i)= & 2 i+1+\lambda v_{n}(i+1, i+1) \\
& +\mu v_{n}(i, i)+\mu v_{n}\left(i+1,(i-1)^{+}\right), \\
v_{n+1}(i, i)= & 2 i+\lambda v_{n}(i+1, i)+\mu v_{n}\left(i,(i-1)^{+}\right)+\mu v_{n}\left(i,(i-1)^{+}\right) .
\end{aligned}
$$

The first term on the RHS of (8a) is larger than the one of $(8 b)$, the second and third terms are ordered by (4) (or equal if $i=0$ ) and the fourth terms are ordered by (3). So $v_{n+1}(i+1, i) \geq v_{n+1}(i, i)$.

Proof of (4)

Case a: $l \geq 2$.

$$
\begin{aligned}
v_{n+1}(i+l, i+1)= & 2 i+l+1+\lambda v_{n}(i+l, i+2) \\
& +\mu v_{n}(i+l-1, i+1)+\mu v_{n}(i+l, i), \\
v_{n+1}(i+l, i)= & 2 i+l+\lambda v_{n}(i+l, i+1) \\
& +\mu v_{n}(i+l-1, i)+\mu v_{n}\left(i+l,(i-1)^{+}\right) .
\end{aligned}
$$

The first term on the RHS of (9a) is larger than the one of $(9 b)$, the other three terms are ordered by (4) (the fourth terms are equal if $i=0$ ).

So $v_{n+1}(i+l, i+1) \geq v_{n+1}(i+l, i)$.

Case b: $l=1$.

$$
\begin{aligned}
v_{n+1}(i+1, i+1)= & 2 i+2+\lambda v_{n}(i+2, i+1) \\
& +\mu v_{n}(i+1, i)+\mu v_{n}(i+1, i), \\
v_{n+1}(i+1, i)= & 2 i+1+\lambda v_{n}(i+1, i+1) \\
& +\mu v_{n}(i, i)+\mu v_{n}\left(i+1,(i-1)^{+}\right) .
\end{aligned}
$$

The first term on the RHS of (10a) is larger than the one of $(10 \mathrm{~b})$, the second and third terms are ordered by (3) and the fourth terms are ordered by (4) (and equal if $i=0)$.

So $v_{n+1}(i+1, i+1) \geq v_{n+1}(i+1, i)$.

\section{Threshold Jockeying}

Let $L(\geq 1)$ be the threshold we consider. If a service completion leads to a difference of $L+1$ between the numbers of jobs in the longest and shortest queue, then one job moves from the longest to the shortest queue. 
The $n$-period costs in the Threshold Jockeying model are denoted by $u_{n}$. It suffices to show that

$$
u_{n}(s) \leq v_{n}(s)
$$

for all $n$ and for all states $s$ that are recurrent in the jockeying model.

The proof follows by induction. For $n=0$ inequality (11) trivially holds. Assuming (11) to hold for $n$ we prove it for $n+1$.

We will distinguish four cases.

Case a: The states $(i+l, i)$ with $l=1, \cdots, L-1$.

$$
\begin{aligned}
u_{n+1}(i+l, i)= & 2 i+l+\lambda u_{n}(i+l, i+1) \\
& +\mu u_{n}(i+l-1, i)+\mu u_{n}\left(i+l,(i-1)^{+}\right), \\
v_{n+1}(i+l, i)= & 2 i+l+\lambda v_{n}(i+l, i+1) \\
& +\mu v_{n}(i+l-1, i)+\mu v_{n}\left(i+l,(i-1)^{+}\right) .
\end{aligned}
$$

So from (11) for $n$ we get $u_{n+1}(i+l, i) \leq v_{n+1}(i+l, i)$.

Case b: The states $(i, i)$.

$$
\begin{aligned}
u_{n+1}(i, i)= & 2 i+\lambda u_{n}(i+1, i) \\
& \left.+\mu u_{n}\left(i,(i-1)^{+}\right)\right)+\mu u_{n}\left(i,(i-1)^{+}\right), \\
v_{n+1}(i, i)= & 2 i+\lambda v_{n}(i+1, i) \\
& \left.+\mu v_{n}\left(i,(i-1)^{+}\right)\right)+\mu v_{n}\left(i,(i-1)^{+}\right) .
\end{aligned}
$$

So $u_{n+1}(i, i) \leq v_{n+1}(i, i)$.

Case c: The states $(i+L, i)$ with $i>0$.

$$
\begin{aligned}
u_{n+1}(i+L, i)= & 2 i+L+\lambda u_{n}(i+L, i+1) \\
& +\mu u_{n}(i+L-1, i)+\mu u_{n}(i+L-1, i), \\
v_{n+1}(i+L, i)= & 2 i+L+\lambda v_{n}(i+L, i+1) \\
& +\mu v_{n}(i+L-1, i)+\mu v_{n}(i+L, i-1) .
\end{aligned}
$$

Comparing the RHS of (14a) and (14b) the only difficult term is the fourth one. But by (2) we have $v_{n}(i+L, i-1) \geq v_{n}(i+L-1, i)$, so (11) gives $u_{n}(i+L-1, i) \leq$ $v_{n}(i+L, i-1)$. Hence $u_{n+1}(i+L, i) \leq v_{n+1}(i+L, i)$ for $i>0$. 
Case d: The state $(L, 0)$.

$$
\begin{aligned}
& u_{n+1}(L, 0)=L+\lambda u_{n}(L, 1)+\mu u_{n}(L-1,0)+\mu u_{n}(L, 0) \\
& v_{n+1}(L, 0)=L+\lambda v_{n}(L, 1)+\mu v_{n}(L-1,0)+\mu v_{n}(L, 0)
\end{aligned}
$$

So (11) for $n$ immediately gives $u_{n+1}(L, 0) \leq v_{n+1}(L, 0)$.

This completes the proof of (11).

\section{CONCLUSION}

The Jockeying model underestimates the mean number of jobs in the system, and hence gives a lower bound for the mean waiting time.

\section{Threshold Blocking}

In the Threshold Blocking model the server in the shortest queue is blocked as long as the difference between the queue lengths in the longest and shortest queue equals the threshold $L$. In order to prove that Threshold Blocking yields an upper bound on the mean waiting time we will show that for all states $s$ that are recurrent for the Threshold Blocking model and for all $n$ we have

$$
v_{n}(s) \leq w_{n}(s)
$$

As said before, $w_{n}$ denotes the $n$-period costs for Threshold Blocking. By definition $v_{0}=w_{0}=0$, so (16) holds for $n=0$. Assuming (16) to hold for $n$ we will prove it for $n+1$.

We will distinguish three cases.

Case a: The states $(i+l, i)$ with $l=1, \cdots, L-1$.

$$
\begin{aligned}
w_{n+1}(i+l, i)= & 2 i+l+\lambda w_{n}(i+l, i+1) \\
& +\mu w_{n}(i+l-1, i)+\mu w_{n}\left(i+l,(i-1)^{+}\right), \\
v_{n+1}(i+l, i)= & 2 i+l+\lambda v_{n}(i+l, i+1) \\
& +\mu v_{n}(i+l-1, i)+\mu v_{n}\left(i+l,(i-1)^{+}\right) .
\end{aligned}
$$

So (16) immediately gives $v_{n+1}(i+l, i) \leq w_{n+1}(i+l, i)$. 
Case b: The states $(i, i)$.

$$
\begin{aligned}
w_{n+1}(i, i)= & 2 i+\lambda w_{n}(i+1, i) \\
& +\mu w_{n}\left(i,(i-1)^{+}\right)+\mu w_{n}\left(i,(i-1)^{+}\right), \\
v_{n+1}(i, i)=2 & +\lambda v_{n}(i+1, i) \\
& +\mu v_{n}\left(i,(i-1)^{+}\right)+\mu v_{n}\left(i,(i-1)^{+}\right) .
\end{aligned}
$$

So $v_{n+1}(i, i) \leq w_{n+1}(i, i)$.

Case c: The states $(i+L, i)$.

$$
\begin{aligned}
w_{n+1}(i+L, i)= & 2 i+L+\lambda w_{n}(i+L, i+1) \\
& +\mu w_{n}(i+L-1, i)+\mu w_{n}(i+L, i), \\
v_{n+1}(i+L, i)= & 2 i+L+\lambda v_{n}(i+L, i+1) \\
& +\mu v_{n}(i+L-1, i)+\mu v_{n}\left(i+L,(i-1)^{+}\right) .
\end{aligned}
$$

Using (16), and for the fourth term (4), we get $v_{n+1}(i+L, i) \leq w_{n+1}(i+L, i)$.

This completes the proof of (16) for $n+1$.

\section{CONCLUSION}

Threshold Blocking gives upper bounds for the mean number of jobs and the mean waiting time in the original shortest queue system.

\section{Stochastic monotonicity}

In sections 4-6 we were interested in the mean number of jobs in the system. Using the same approach one may establish the stochastic monotonicity of the total number of jobs in the system. Let $M$ be any integer, and define the cost function $c(s)=1$ if $\Sigma s_{i} \geq M$ and 0 otherwise. It is easily seen that for this cost function the monotonicity results in sections $4-6$ hold as well. So, writing $F_{T J}(i), F_{S Q}(i)$ and $F_{T B}(i)$ for the probability of having at most $i$ jobs in the jockeying system, the standard shortest queue system and the blocking system respectively, we have for all $i$

$$
F_{T J}(i) \geq F_{S Q}(i) \geq F_{T B}(i)
$$




\section{Analyzing the two threshold models}

So far we have shown that the mean number of jobs and the mean waiting time of the shortest queue model can be bounded between the corresponding quantities of two threshold models. In this section it will be shown that the two threshold models are easier to evaluate than the shortest queue model.

From now on we consider the general situation with $N(\geq 2)$ servers again. The states of the two threshold models are characterized by the vectors $s=\left(s_{1}, \cdots, s_{N}\right)$ where by symmetry we may assume that $s_{1} \geq \cdots \geq s_{N}$. So $s_{1}$ is the length of the longest queue, $s_{2}$ is the length of the second longest queue, and so on. The analysis can be restricted to the recurrent states, which are the ones with $s_{1} \leq s_{N}+L$. This form of state space suggests to use the matrix-geometric approach, as developed by Neuts [22]. Indeed, it appears that this approach is very well suited for the analysis of the two models. The advantage of this approach is that the problem of solving infinitely many equilibrium equations is reduced to that of solving finitely many.

\subsection{THRESHOLD BLOCKING}

We first consider the model with Threshold Blocking. Application of the matrix-geometric approach requires a partitioning of the state space. Let us first define level $l$ as the set of states $s$ with $s_{1}=l$. Then we partition the state space into the levels $0,1, \cdots, L, L+1, \cdots$ and put together the levels $0,1, \cdots, L-1$ with less regular behaviour in one set. The states at each level may be ordered lexicographically. For this partitioning the generator $Q$ is of the form

$$
Q=\left(\begin{array}{cccccc}
B_{00} & B_{01} & 0 & 0 & 0 & \ldots \\
B_{10} & B_{11} & A_{0} & 0 & 0 & \ldots \\
0 & A_{2} & A_{1} & A_{0} & 0 & \ldots \\
0 & 0 & A_{2} & A_{1} & A_{0} & \ldots \\
\vdots & \vdots & \vdots & \ddots & \ddots & \ddots
\end{array}\right) .
$$

The blocks $A_{0}, A_{1}$ and $A_{2}$ are of order $m \times m$, where $m$ is the number of states at each of the levels $l, l \geq L$, so

$$
m=\left(\begin{array}{c}
N+L-1 \\
L
\end{array}\right)
$$

The Markov process $Q$ is irreducible and, since two states at levels $>L$ can reach each other via paths not passing through levels $\leq L$, the generator $A_{0}+A_{1}+A_{2}$ is also irreducible. 
So theorem 1.7.1 in Neuts [22] can readily be applied. As mentioned in section 2, the condition $N \mu>\lambda$ is no longer sufficient for ergodicity, because blocking reduces the service capacity, but the desired condition is given by theorem 1.7.1 stating that $Q$ is ergodic if and only if

$$
\pi A_{0} e<\pi A_{2} e
$$

where $e$ is the column vector of ones and $\pi$ is the solution of

$$
\pi\left(A_{0}+A_{1}+A_{2}\right)=0, \quad \pi e=1 .
$$

By partitioning the equilibrium probability vector $p$ into the vector $\left(p_{0}, \cdots, p_{L-1}\right)$ and into the sequence of vectors $p_{L}, p_{L+1}, \cdots$ where $p_{l}$ is the equilibrium probability vector of level $l$, we conclude from the same theorem that if $Q$ is ergodic, then

$$
p_{l}=p_{L} R^{l-L}, \quad l>L,
$$

where the matrix $R$ is the minimal nonnegative solution of the matrix quadratic equation

$$
A_{0}+R A_{1}+R^{2} A_{2}=0
$$

For our choice of partitioning the state space the matrix $A_{0}$ has a special structure which can be exploited to determine $R$ efficiently. Since it is only possible to jump from level $l$ to level $l+1$ via state $(l, \cdots, l)$, it follows that all rows of $A_{0}$ are zero, except for the last row. Thus $A_{0}$ is of the form

$$
A_{0}=\left(\begin{array}{l}
0 \\
x
\end{array}\right)
$$

where the vector $x=\left(x_{1}, \cdots, x_{m}\right)$. Since the rows in $R$ corresponding to the zero rows in $A_{0}$, are also zero (see e.g. the proof of theorem 1.3.4 in Neuts [22]), we conclude that $R$ is also of the form

$$
R=\left(\begin{array}{l}
0 \\
y
\end{array}\right)
$$

where the vector $y=\left(y_{1}, \cdots, y_{m}\right)$. By insertion of the special forms of $R$ and $A_{0}$, eq. (22) simplifies to the following set of quadratic equations

$$
x+y\left(A_{1}+y_{m} A_{2}\right)=0
$$


or equivalently

$$
y=x+y\left(I+A_{1}+y_{m} A_{2}\right)
$$

where $I$ is the $m \times m$ identity matrix. Equations (25) can be solved efficiently by successive substitutions starting with $y=0$ (cf. p. 9 in [22]). The number of iterations required to compute $y$ sufficiently accurate can be reduced considerably (roughly halved) by using the Gauss-Seidel technique.

Above, we showed that the special structure of $A_{0}$ implies a special structure of $R$. In fact, in the more general case that $A_{0}$ is given by

$$
A_{0}=a \cdot b,
$$

where $a$ is a column vector and $b$ is a row vector, Ramaswami and Latouche [23] show that $R$ is of the form

$$
R=a \cdot y,
$$

where the row vector $y$ satisfies (cf. (24))

$$
b+y\left(A_{1}+(y a) A_{2}\right)=0 .
$$

By substituting the form (23) into (21) the matrix-geometric solution simplifies to

$$
p_{l}=y_{m}^{l-L-1} p(L, \cdots, L) y, \quad l>L .
$$

The remaining probability vectors $p_{L}, p_{L-1}, \ldots, p_{0}$ can be solved from the boundary conditions. In fact, again by exploiting the property that it is only possible to jump from level $l$ to level $l+1$ via state $(l, \cdots, l)$, it is easily verified that these vectors can be solved recursively from the equilibrium equations for the levels $L$, $L-1, \cdots, 0$. The solution of the boundary conditions is worked out in more detail in subsection 8.3.

\subsection{THRESHOLD JOCKEYING}

The model with Threshold Jockeying can be treated in exactly the same way. In addition, for this model it can be shown that $N \mu>\lambda$ is necessary and sufficient for ergodicity (see Adan et al. [2]) and the component $y_{m}$ can be found explicitly by using the following balance argument.

Let $V_{l}$ be the set of states with $s_{1}+\cdots+s_{N}=l$ and $P\left(V_{l}\right)$ be the equilibrium probability for the set $V_{l}$. By balancing the flow between the sets $V_{l}$ and $V_{l+1}$ it follows that for all $l>(N-1) L$,

$$
P\left(V_{l+1}\right) N \mu=P\left(V_{l}\right) \lambda,
$$


and by applying this relation $N$ times, we get

$$
P\left(V_{l+N}\right)=\left(\frac{\lambda}{N \mu}\right)^{N} P\left(V_{l}\right)
$$

On the other hand, (26) implies that $p\left(s_{1}+1, \cdots, s_{N}+1\right)=y_{m} p\left(s_{1}, \cdots, s_{N}\right)$ if $s_{1}>L$, so it follows that for all $l>N L$

$$
P\left(V_{l+N}\right)=y_{m} P\left(V_{l}\right)
$$

Combining (27) and (28) yields

$$
y_{m}=\left(\frac{\lambda}{N \mu}\right)^{N}
$$

Substitution of (29) into (25) leads to a set of linear equations for the other components of the vector $y$. Note that these equations form a contraction scheme. A convenient method to solve such schemes is the iteration method presented in Van der Wal and Schweitzer [27]. This method has the advantage that it provides upper and lower bounds for the vector $y$.

\subsection{SOLVING THE BOUNDARY CONDITIONS}

In this section we show how the probability vector $p_{l}$ may be found given the solution at the levels $>l$. Up to some multiplicative constant $p_{l}$ equals the equilibrium probability vector of the Markov process restricted to level $l$, i.e. excursions to other levels are not considered. So if we compute the equilibrium probability vector of the restricted Markov process, then proper normalization of this probability vector produces $p_{l}$.

Let $q(a, b)$ be the transition rate from state $a$ to state $b$ for the original Markov process and let $r(a, b)$ be the one for the restricted Markov process. The rate $r(a, b)$ is given by $q(a, b)$ plus the rate due to excursions to other levels starting in $a$ and ending in $b$. An excursion to levels $<l$ always ends in $(l, l-1, \cdots, l-1)$ and an excursion to levels $>l$ always starts at $(l, \cdots, l)$ and ends with the probability $e(b)$ in state $b$ at level $l$ where $e(b)$ is given by

$$
e(b)=\frac{\sum_{s_{1}=l+1} p(s) q(s, b)}{\sum_{s_{1}=l+1} p(s) \sum_{a_{\mathrm{l}}=l} q(s, a)}
$$


Hence, for two states $a$ and $b$ at level $l$ we get

$$
\begin{aligned}
r(a, b)= & q(a, b) \\
& +\left\{\begin{array}{ll}
\sum_{s_{1}=l-1} q(a, s) & \text { if } b=(l, l-1, \cdots, l-1) \\
0 & \text { otherwise }
\end{array}\right\}+\left\{\begin{array}{ll}
\lambda e(b) & \text { if } a=(l, \cdots, l) \\
0 & \text { otherwise }
\end{array}\right\} .
\end{aligned}
$$

Thus the transition rates $r(a, b)$ can be obtained once the (unnormalized) probabilities $p(s)$ for states $s$ at level $l+1$ are known. Then the equilibrium probability vector of the Markov process restricted to level $l$ can readily be computed using the iteration method in [27]. As mentioned before, this iteration method has the advantage that it provides good upper and lower bounds for the equilibrium distribution. To produce $p_{l}$, we normalise the probability vector of the restricted Markov process using the equation stating that the flow from level $l$ to $l+1$ is equal to the flow from level $l+1$ to $l$, i.e.

$$
p(l, \cdots, l) \lambda=\sum_{s_{1}=l+1} p(s) \sum_{a_{1}=l} q(s, a) .
$$

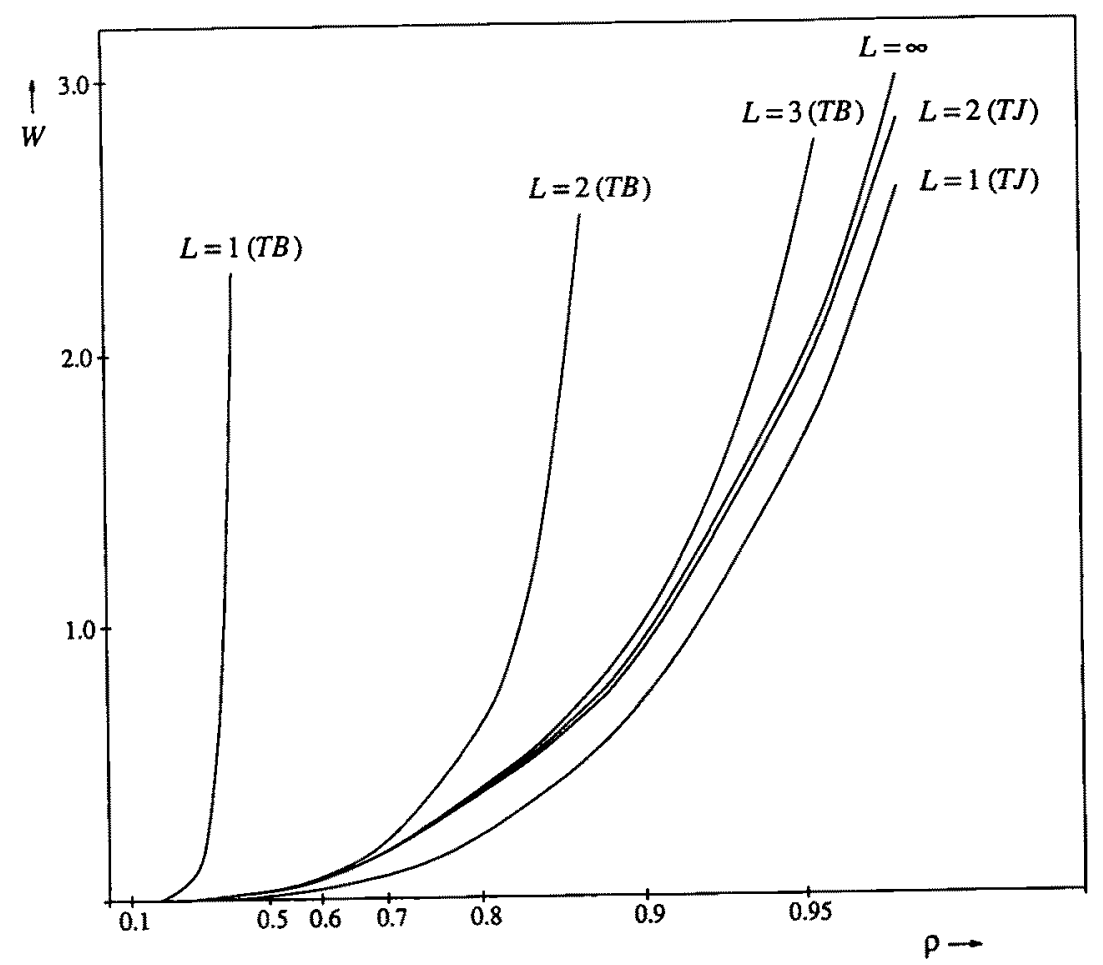

Fig. 1. Bounds for the normalized mean waiting time $W$ for the case of 10 servers. 
Starting with $p_{L+1}$ given by (26), we can use the approach sketched above to recursively compute the vectors $p_{L}, \cdots, p_{0}$.

\section{Numerical results}

This section is devoted to some numerical results. For a system with 10 parallel servers we depict in fig. 1 for increasing values of the threshold $L$ the upper and lower bounds for the "normalized" mean waiting time $W$ as a function of the workload $\rho$ defined by

$$
\rho=\frac{\lambda}{N \mu}
$$

(The normalized mean waiting time $W$ is defined as the quotient of the mean waiting time and the mean service time.)

We used a logarithmic axis for $\rho$ in order to blow up the relevant region near $\rho=1$. In table 1 we list the normalized mean waiting times corresponding to the curves in fig. 1 . If for a combination of $\rho$ and $L$ the blocking model is not ergodic, then the upper bound produced by the blocking model is infinite.

As $L$ tends to infinity, then the upper and lower bounds can be expected to converge to the mean waiting time of the shortest queue system. Figure 1

Table 1

Normalized mean waiting times corresponding to the curves in fig. 1.

\begin{tabular}{|c|c|c|c|c|c|c|c|}
\hline \multirow{3}{*}{$\begin{array}{l}N=10 \\
\rho\end{array}$} & \multicolumn{7}{|c|}{ Mean waiting time/mean service time } \\
\hline & \multicolumn{3}{|c|}{ Threshold Jockeying } & \multirow[b]{2}{*}{$L=\infty$} & \multicolumn{2}{|c|}{ Threshold Blocking } & \multirow[b]{2}{*}{$L=1$} \\
\hline & $L=1$ & $L=2$ & $L=3$ & & $L=3$ & $L=2$ & \\
\hline 0.1 & 0.000 & 0.000 & 0.000 & 0.000 & 0.000 & 0.000 & 0.000 \\
\hline 0.2 & 0.000 & 0.000 & 0.000 & 0.000 & 0.000 & 0.000 & 0.001 \\
\hline 0.3 & 0.000 & 0.001 & 0.001 & 0.001 & 0.001 & 0.001 & 0.052 \\
\hline 0.4 & 0.001 & 0.006 & 0.006 & 0.006 & 0.006 & 0.006 & 1.003 \\
\hline 0.5 & 0.007 & 0.025 & 0.025 & 0.025 & 0.025 & 0.025 & $\infty$ \\
\hline 0.6 & 0.025 & 0.069 & 0.069 & 0.069 & 0.069 & 0.071 & $\infty$ \\
\hline 0.7 & 0.075 & 0.172 & 0.174 & 0.174 & 0.174 & 0.198 & $\infty$ \\
\hline 0.8 & 0.218 & 0.391 & 0.400 & 0.400 & 0.404 & 0.627 & $\infty$ \\
\hline 0.85 & 0.362 & 0.558 & 0.575 & 0.576 & 0.592 & 1.434 & $\infty$ \\
\hline 0.9 & 0.669 & 0.878 & 0.910 & 0.913 & 0.988 & 5.083 & $\infty$ \\
\hline 0.925 & 0.993 & 1.219 & 1.261 & 1.267 & 1.455 & $\infty$ & $\infty$ \\
\hline 0.95 & 1.651 & 1.894 & 1.948 & 1.957 & 2.564 & $\infty$ & $\infty$ \\
\hline 0.96 & 2.148 & 2.397 & 2.456 & 2.468 & 3.606 & $\infty$ & $\infty$ \\
\hline 0.97 & 2.978 & 3.234 & 3.298 & 3.312 & 5.962 & $\infty$ & $\infty$ \\
\hline 0.98 & 4.641 & 4.903 & 4.974 & 4.991 & 16.428 & $\infty$ & $\infty$ \\
\hline 0.99 & 9.637 & 9.906 & 9.983 & 10.003 & $\infty$ & $\infty$ & $\infty$ \\
\hline
\end{tabular}


Table 2

Bounds for the normalized mean waiting time.

\begin{tabular}{|c|c|c|c|c|c|c|}
\hline$\rho$ & $N$ & $L$ & $m$ & $W_{T J}$ & $W_{T B}$ & $W_{M / M / N}$ \\
\hline \multirow[t]{11}{*}{0.80} & 2 & 6 & 7 & 1.955 & 1.959 & 1.778 \\
\hline & 5 & 4 & 70 & 0.754 & 0.758 & 0.554 \\
\hline & 10 & 3 & 220 & 0.355 & 0.359 & 0.205 \\
\hline & 15 & 3 & 680 & 0.220 & 0.221 & 0.106 \\
\hline & 20 & 3 & 1540 & 0.151 & 0.151 & 0.064 \\
\hline & 25 & 2 & 325 & 0.110 & 0.116 & 0.042 \\
\hline & 30 & 2 & 465 & 0.083 & 0.085 & 0.029 \\
\hline & 35 & 2 & 630 & 0.064 & 0.064 & 0.021 \\
\hline & 40 & 2 & 820 & 0.050 & 0.050 & 0.015 \\
\hline & 45 & 2 & 1035 & 0.040 & 0.040 & 0.011 \\
\hline & 50 & 2 & 1275 & 0.032 & 0.032 & 0.009 \\
\hline \multirow[t]{11}{*}{0.90} & 2 & 7 & 8 & 4.474 & 4.483 & 4.263 \\
\hline & 5 & 5 & 126 & 1.797 & 1.805 & 1.525 \\
\hline & 10 & 4 & 715 & 0.913 & 0.917 & 0.669 \\
\hline & 15 & 4 & 3060 & 0.616 & 0.616 & 0.402 \\
\hline & 20 & 3 & 1540 & 0.463 & 0.469 & 0.275 \\
\hline & 25 & 3 & 2925 & 0.370 & 0.372 & 0.203 \\
\hline & 30 & 3 & 4960 & 0.306 & 0.307 & 0.157 \\
\hline & 35 & 3 & 7770 & 0.260 & 0.260 & 0.126 \\
\hline & 40 & 3 & 11480 & 0.224 & 0.224 & 0.103 \\
\hline & 45 & 3 & 16215 & 0.195 & 0.195 & 0.086 \\
\hline & 50 & 3 & 22100 & 0.172 & 0.172 & 0.073 \\
\hline \multirow[t]{11}{*}{0.95} & 2 & 9 & 10 & 9.487 & 9.491 & 9.256 \\
\hline & 5 & 6 & 210 & 3.827 & 3.836 & 3.511 \\
\hline & 10 & 5 & 2002 & 1.957 & 1.961 & 1.651 \\
\hline & 15 & 4 & 3060 & 1.335 & 1.344 & 1.049 \\
\hline & 20 & 4 & 8855 & 1.022 & 1.025 & 0.755 \\
\hline & 25 & 4 & 20475 & 0.833 & 0.834 & 0.583 \\
\hline & 30 & 4 & 40920 & 0.727 & 0.727 & 0.470 \\
\hline & 35 & 3 & 7770 & 0.613 & 0.621 & 0.390 \\
\hline & 40 & 3 & 11480 & 0.542 & 0.547 & 0.332 \\
\hline & 45 & 3 & 16215 & 0.486 & 0.489 & 0.287 \\
\hline & 50 & 3 & 22100 & 0.441 & 0.442 & 0.252 \\
\hline
\end{tabular}

demonstrates that the bounds are tight for already small values of $L$ and that in order to produce bounds with the same accuracy the threshold $L$ for the blocking model is larger than the one for the jockeying model. Apparently, temporarily switching off the servers in the blocking model has a more important effect on the mean waiting time than moving work from one queue to another in the jockeying model. An intuitive explanation may be that blocking directly decreases the capacity, whereas jockeying may be later on leads to a more efficient use of the capacity. 
In table 2 we list for systems with 2 up to 50 parallel servers the normalized mean waiting time for the Threshold Jockeying model $\left(W_{T J}\right)$ and the Threshold Blocking model $\left(W_{T B}\right)$ for increasing values of $\rho$. For both models we used the same threshold $L$. The threshold $L$ in table 2 is the smallest one for which the gap between the normalized mean waiting time of the upper and lower bound model is less than 0.01 .

Table 2 illustrates that the bounds for the mean waiting time are tight for already small values of $L$ and that larger systems require smaller thresholds to produce the mean waiting time with the same accuracy.

For comparison, we list in table 2 the normalized mean waiting time $W_{M / M / N}$ for the $M / M / N$ queue with arrival rate $\lambda$ and service rate $\mu$ for each server. Note that the mean waiting time for the $M / M / N$ system is identical to the one for the Threshold Jockeying model with $L=1$. The $M / M / N$ system may be used as a simple approximation for the shortest queue system. However, table 2 demonstrates that this approximation performs poorly compared to the Threshold Jockeying model with $L$ somewhat larger than one.

The main numerical effort to solve the upper and lower bound model consists of solving eqs. (25) and the equilibrium equations at the highest level $L$. Both sets of equations consists of $m$ equations. These equations are solved by iteration methods (see section 8). Empirically we have found that the number of iterations required to solve these equations sufficiently accurate is proportional to (roughly) $N /(1-\rho)$. The amount of work in each iteration is proportional to the number of equations and the number of nonzero coefficients in each equation. A nonzero coefficient corresponds to a transition possibility from one state to another. So, on average, the number of nonzero coefficients in an equation is given by 1 (an arrival) plus $\min (N, L+1)$ (a service completion). Hence we may conclude that the amount of work required to solve the upper and lower bound model is proportional to the product of $m$ and $1+\min (N, L+1)$ and $N /(1-\rho)$. Recall that

$$
m=\left(\begin{array}{c}
N+L-1 \\
L
\end{array}\right)
$$

So $m$ increases very fast in $N$ and $L$. To give an impression of computation times we present in table 3 for some of the problems listed in table 2 the computation times on a SUN workstation. Of course, one should keep in mind that computation times highly depend on the implementation and hardware.

\section{Comparison with simple truncation}

Alternatively the shortest queue problem can be approximated by simple state space truncation, i.e. by treating all queues as bounded. In this approximation arriving jobs finding all buffers full are rejected and thus receive zero waiting times. Using the Markov reward technique exposed in the present paper it is readily 
Table 3

Computation times on a SUN workstation for some of the problems in table 2.

\begin{tabular}{lllrl}
\hline$\rho$ & $N$ & $L$ & $m$ & $\begin{array}{l}\text { Computation } \\
\text { time (h:min:sec) }\end{array}$ \\
\hline 0.8 & 10 & 3 & 220 & $0: 00: 06$ \\
0.9 & 10 & 4 & 715 & $0: 00: 32$ \\
0.9 & 25 & 3 & 2925 & $0: 05: 34$ \\
0.9 & 50 & 3 & 22100 & $1: 31: 29$ \\
0.95 & 5 & 6 & 210 & $0: 00: 08$ \\
0.95 & 10 & 5 & 2002 & $0: 03: 03$ \\
0.95 & 20 & 4 & 8855 & $0: 24: 48$ \\
0.95 & 30 & 4 & 40920 & $2: 54: 53$ \\
\hline
\end{tabular}

verified that this approximation provides a lower bound for the waiting time in the shortest queue problem.

To compare the performance of simple truncation with the use of the two threshold models we list in table 4 for a system with 5 servers the normalized mean waiting time for simple truncation $\left(W_{S T}\right)$ and the ones for the shortest queue system and the two threshold models for increasing values of the workload $\rho$. For the simple truncation model $L$ denotes the capacity of each queue (including the job in service). Recall that $W_{T J}$ and $W_{T B}$ are the normalized mean waiting times for the threshold models and $W$ is the normalized mean waiting time for the original shortest queue problem.

Table 4 illustrates that especially in heavy load simple truncation performs poorly compared to the upper and lower bounds produced by the threshold models. Simple truncation can of course be improved, for instance by exploiting that the probability mass concentrates around the diagonal. However more sophisticated approximations still have the disadvantage that no error bounds can be given.

Table 4

Simple approximation and bounds for the normalized mean waiting time.

\begin{tabular}{|c|c|c|c|c|c|c|c|c|}
\hline \multicolumn{2}{|c|}{$N=5$} & \multicolumn{3}{|c|}{ Simple truncation } & \multicolumn{4}{|c|}{ Threshold models } \\
\hline$\rho$ & $W$ & $L$ & $m$ & $W_{S T}$ & $L$ & $m$ & $W_{T J}$ & $W_{T B}$ \\
\hline 0.5 & 0.109 & 2 & 15 & 0.100 & 2 & 15 & 0.107 & 0.116 \\
\hline 0.6 & 0.237 & 3 & 35 & 0.232 & 3 & 35 & 0.237 & 0.238 \\
\hline 0.7 & 0.405 & 4 & 70 & 0.397 & 3 & 35 & 0.404 & 0.412 \\
\hline 0.8 & 0.754 & 7 & 330 & 0.749 & 4 & 70 & 0.754 & 0.758 \\
\hline 0.9 & 1.798 & 15 & 3876 & 1.790 & 5 & 126 & 1.797 & 1.805 \\
\hline 0.95 & 3.827 & 33 & 66045 & 3.819 & 6 & 210 & 3.827 & 3.836 \\
\hline
\end{tabular}




\section{Conclusion}

As we have seen, it is possible to derive tight bounds on the mean waiting time in the shortest queue system by comparing it with two similar systems that are easier to analyse: the shortest queue system with Threshold Jockeying and the one with Threshold Blocking. Furthermore, we can get bounds for the distribution of the total number of jobs in the system.

These results may be used to obtain insight in the behaviour of shortest queue routing. Exploiting this insight it should be possible to produce fairly simple approximations for shortest queue stations in queueing networks.

\section{References}

[1] I.J.B.F. Adan, J. Wessels and W.H.M. Zijm, Analysis of the symmetric shortest queue problem, Stochastic Mod. 6 (1990) 691-713.

[2] I.J.B.F. Adan, J. Wessels and W.H.M. Zijm, Matrix-geometric analysis of the shortest queue problem with threshold jockeying, Oper. Res. Lett. 13 (1993) 107-112.

[3] J.P.C. Blanc, The power-series algorithm applied to the shortest-queue model, Oper. Res. 40 (1992) $157-167$.

[4] J.W. Cohen and O.J. Boxma, Boundary Value Problems in Queueing System Analysis (NorthHolland, Amsterdam, 1983).

[5] B.W. Conolly, The autostrada queueing problem, J. Appl. Prob. 21 (1984) 394-403.

[6] N.M. van Dijk and B.F. Lamond, Simple bounds for finite single-server exponential tandem queues, Oper. Res. 36 (1988) 470-477.

[7] N.M. van Dijk and J. van der Wal, Simple bounds and monotonicity results for finite multi-server exponential tandem queues, Queueing Syst. 4 (1989) 1-16.

[8] G. Fayolle and R. Iasnogorodski, Two coupled processors: the reduction to a Riemann-Hilbert problem, Z. Wahrsch. Verw. Gebiete (1979) 325-351.

[9] L. Flatto and H.P. McKean, Two parallel queues with equal servicing rates, Science Report, RC5916, IBM (1977).

[10] L. Flatto and H.P. McKean, Two queues in parallel, Comm. Pure Appl. Math. 30 (1977) 255-263.

[11] G.J. Foschini and J. Salz, A basic dynamic routing problem and diffusion, IEEE Trans. Commun. COM-26 (1978) 320-327.

[12] I. Gertsbakh, The shorter queue problem: A numerical study using the matrix-geometric solution, Eur. J. Oper. Res. 15 (1984) 374-381.

[13] W.K. Grassmann, Transient and steady state results for two parallel queues, OMEGA Int. J. Manag. Sci. 8 (1980) 105-112.

[14] W.K. Grassmann and Y. Zhao, A numerically stable algorithm for two server queue models, Queueing Syst. 8 (1991) 59-80.

[15] F.A. Haight, Two queues in parallel, Biometrica 45 (1958) 401-410.

[16] S. Halfin, The shortest queue problem, J. Appl. Prob. 22 (1985) 865-878.

[17] G. Hooghiemstra, M. Keane and S. van de Ree, Power series for stationary distributions of coupled processor models, SIAM J. Appl. Math. (1988) 1159-1166.

[18] J.F.C. Kingman, Two similar queues in parallel, Ann. Math. Statist. 32 (1961) 1314-1323.

[19] C. Knessl, B.J. Matkowsky, Z. Schuss and C. Tier, Two parallel queues with dynamic routing, IEEE Trans. Commun. COM-34 (1986) 1170-1175.

[20] J.C.S. Lui and R.R. Muntz, Algorithmic approach to bounding the mean response time of a minimum expected delay routing system, Perf. Eval. Rev. 20 (1992) 140-151. 
[21] R.D. Nelson and T.K. Philips, An approximation to the response time for shortest queue routing, Perf. Eval. Rev. 17 (1989) 181-189.

[22] M.F. Neuts, Matrix-Geometric Solutions in Stochastic Models (Johns Hopkins University Press, Baltimore, 1981).

[23] V. Ramaswami and G. Latouche, A general class of Markov processes with explicit matrixgeometric solutions, OR Spectrum 8 (1986) 209-218.

[24] B.M. Rao and M.J.M. Posner, Algorithmic and approximation analysis of the shorter queue model, Naval Res. Log. 34 (1987) 381-398.

[25] R. Schassberger, Ein Wartesystem mit zwei parallelen Warteschlängen, Computing 3 (1968) 110 124.

[26] R. Schassberger, A service system with two parallel queues, Computing 4 (1969) 24-29.

[27] J. van der Wal and P.J. Schweitzer, Iterative bounds on the equilibrium distribution of a finite Markov chain, Prob. Eng. Inf. Sci. 1 (1987) 117-131.

[28] J. van der Wal, Monotonicity of the throughput of a closed exponential queueing network in the number of jobs, OR Spectrum 11 (1989) 97-100. 